\title{
Advanced laser sources for Sentinel-5/UVNS instrument calibration
}

Matthew Edgar, John Nicholls, Gareth Maker, David Mesquita, Graeme Malcom

Matthew P. Edgar, John Nicholls, Gareth Maker, David Mesquita, Graeme Malcom, "Advanced laser sources for Sentinel-5/UVNS instrument calibration," Proc. SPIE 11852, International Conference on Space Optics ICSO 2020, 1185237 (11 June 2021); doi: 10.1117/12.2599535

SPIE Event: International Conference on Space Optics - ICSO 2021, 2021, Online Only 


\section{International Conference on Space Optics-ICSO 2020}

Virtual Conference

30 March-2 April 2021

Edited by Bruno Cugny, Zoran Sodnik, and Nikos Karafolas
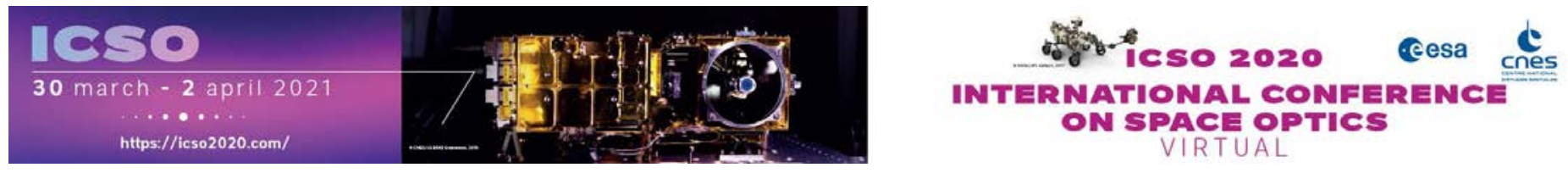

\section{Advanced laser sources for Sentinel-5/UVNS instrument calibration}

\section{Cesa issopoceatings lecnes}




\title{
Advanced laser sources for Sentinel-5/UVNS instrument calibration
}

\author{
Matthew Edgar ${ }^{\mathrm{a}}$, John Nicholls ${ }^{\mathrm{a}}$, Gareth Maker ${ }^{\mathrm{a}}$, David Mesquita ${ }^{\mathrm{b}}$, and Graeme Malcolm ${ }^{\mathrm{a}}$ \\ ${ }^{a}$ M Squared Lasers, 1 Kelvin Campus, West of Scotland Science Park, Glasgow, G20 0SP, \\ United Kingdom

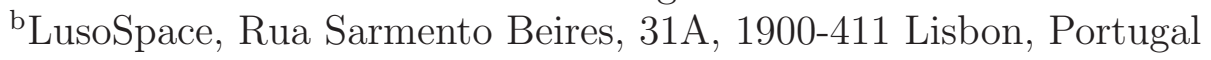

\begin{abstract}
The Sentinel-5/UVNS instrument is a spectrometer developed within the Copernicus program for monitoring trace gas concentrations and aerosols in Earth's atmosphere with unprecedented accuracy and resolution. The performance of the satellite spectrometers in orbit relies upon accurate characterisation and radiometric calibration of the optical ground support equipment (OGSE). M Squared Lasers has developed a series of advanced tuneable, fully-automated, remotely controlled, narrow-linewidth laser systems, providing rapid scanning across the extensive wavelength operating range from 260-2700 nm, with a number of unique features that ensure stringent source requirements are met throughout the mission lifetime. To the best of our knowledge, these laser systems are the first of their kind, demonstrating a significant leap in laser technology with regards to remote controlled automated operation, gapless wavelength coverage from UV to SWIR, functionality and performance. This paper will detail each of the S5 laser sources, describe the engineering achievements in their development.
\end{abstract}

Keywords: Sentinel 5, Calibration, Laser System, Laser Development

\section{INTRODUCTION}

Our planet is changing as a result of human activity and many of the climate change indicators can only be monitored from space. Next generation space instruments studying the atmosphere require wavelength coverage from the deep UV to the SWIR spectral regions to be able to study these critical climate indicators through their atmospheric absorptions.

M Squared's SolsTiS laser platform is the only system in the world able to provide the required broad spectral coverage and the ability to automatically step through 1000nm's with pm resolution at high speed - ensuring instruments are calibrated accurately and at high speed, limiting the cost of test campaigns in expensive thermal vacuum chambers.

M Squared Lasers have gained a successful track record developing advanced laser systems with unrivalled performance to support the instrument calibration of important space missions, including Microcarb, Sentinel 4, Sentinel 5P, and most recently Sentinel 5 .

Shortly before M Squared's were awarded the contract to develop the sources for Sentinel 5, a team of specialist laser engineers worked closely with ESA, Airbus DS, and KNMI on the design and implementation of fully automated high-speed tuneable source covering $580-1000 \mathrm{~nm}$ to re-calibrate the TROPOMI spectrometer on-board the fully-assembled S5P flight model. The TROPOMI spectrometer is used to monitor air quality, ozone and surface UV-B, providing trace gas concentrations in the atmosphere for $\mathrm{NO} 2, \mathrm{SO} 2, \mathrm{CO}, \mathrm{CH} 4, \mathrm{CH} 2 \mathrm{O}$, $\mathrm{C} 2 \mathrm{H} 2 \mathrm{O} 2$, BRO2, clouds and aerosol. TROPOMI had already been calibrated using lasers from another vendor that did not meet the performance requirements, and unfortunately was found to be dysfunctional in certain wavebands shortly before launch date. The spectacular earth observation data ${ }^{1}$ that Sentinel-5P is now delivering to help monitor our environment would not have been possible without the rapid support from M Squared Lasers overcome this urgent calibration challenge. Indeed the engineering team has been proud to see our awardwinning technology become trusted tools for important space missions to observe and map a multitude of critical atmospheric pollutants that impact our health and affect our climate.

Further author information: (Send correspondence to M.E.)

M.E.: E-mail: matt.edgar@m2lasers.com, Telephone: +44 1419450500 


\section{SENTINEL-5}

ESA is developing a series of next-generation Earth observation missions, on behalf of the joint ESA/European Commission initiative Copernicus. ${ }^{2}$ The Sentinel-5 (S5) mission/satellite will monitor trace gas concentrations and aerosols in the atmosphere: $\mathrm{O} 3, \mathrm{NO} 2, \mathrm{SO} 2, \mathrm{HCHO}, \mathrm{CO}, \mathrm{CH} 4$ and aerosol at high spatial resolution and with a daily global revisit time. Focused on air quality and the interaction between climate and atmospheric composition the mission will provide key information on atmospheric variables in support of European policies for the benefit of European citizens.

Airbus DS is the prime contractor for the S5 satellite. The S5 Optical Ground Support Equipment (OSGE) contract was awarded to Lusospace. S5 has two telescopes and two OGSE systems to allow parallel testing, thus limiting overall on-ground testing campaign duration. Two laser systems a required to feed both OGSE's. High resolution ISRF (instrument spectral response function) measurements will be performed across the ultraviolet to shortwave infrared range within five different spectral bands referred to as the C\&C section: UV1 (270-310nm), U2V (300-500nm), NIR (685-783nm), SWIR1 (1590-1675nm) and SWIR3 (2305-2385nm). The "US" telescope covers UV1, SWIR1 and SWIR3 bands; the second "VN" telescope covers the U2V and NIR bands. There is also a need for "straylight" measurements (SM) at in-between and adjacent wavelength regions of the Sentinel-5 instrument across the wavelength range from $265 \mathrm{~nm}$ to $2700 \mathrm{~nm}$. Specifically, the "US" telescope requires SM from 265-2700nm; the "VN" telescope requires SM from 265-1100 nm.

\section{SOURCE REQUIREMENTS FOR SENTINEL-5/UVNS INSTRUMENT CALIBRATION}

A comprehensive list of the key source requirements is provided in Table 1.

Table 1. Key requirements of the laser sources for Sentinel-5/UVNS instrument calibration across the spectral bands $\mathrm{UV}^{1}{ }^{1}, \mathrm{U}_{2} \mathrm{~V}^{2}, \mathrm{NIR}^{3}, \mathrm{SWIR}^{4}, \mathrm{SWIR}^{5}$ and $\mathrm{STRAY}^{6}$

\begin{tabular}{|c|c|c|}
\hline Requirements & AIT Laser Source Value & C\&C Laser Source Value \\
\hline Spectral Bands & UV1, U2V, NIR & UV1, U2V, NIR, SWIR1, SWIR3, STRAY \\
\hline Spectral Tuning & $260-500 \mathrm{~nm}, 685-770 \mathrm{~nm}$ (discrete) & $260-2700 \mathrm{~nm}$ (continuous) \\
\hline Linewidth & $\leq 2 \mathrm{pm}^{1}, \leq 3 \mathrm{pm}^{2}, \leq 5 \mathrm{pm}^{3}$ & $\leq 2 \mathrm{pm}^{1,4,5}, \leq 3 \mathrm{pm}^{2}, \leq 5 \mathrm{pm}^{3}$ \\
\hline Frequency Stability & $\leq 2 \mathrm{pm} / \mathrm{h}$ & $\leq 2 \mathrm{pm} / 10 \min ^{1,2,3}, \leq 0.5 \mathrm{pm} / 10 \mathrm{~min}^{4,5}$ \\
\hline Wavelength Accuracy & $<5 \mathrm{pm}$ & $<10 \mathrm{pm}^{1},<5 \mathrm{pm}^{2,3},<1 \mathrm{pm}^{4,5}$ \\
\hline Tune Time & $\leq 60 \mathrm{~s}$ & $\leq 30 \mathrm{~s}$ \\
\hline Spectral Purity & $\geq 40 \mathrm{~dB}$ & $\geq 50 \mathrm{~dB}$ \\
\hline Optical Power (Fibre) & $\geq 7 \mathrm{~mW}^{1,2}, \geq 50 \mathrm{~mW}^{3}$ & $\geq 20 \mathrm{~mW}^{1,2}, \geq 100 \mathrm{~mW}^{3}, \geq 5 \mathrm{~mW}^{4}, \geq 2.5 \mathrm{~mW}^{5}$ \\
\hline Power Stability & $<1 \% \mathrm{pkpk} / 10 \mathrm{~min}$ & $<1 \% \mathrm{RMS} / 24 \mathrm{hr}$ \\
\hline Power Control & $50 \mathrm{~dB}$ & $60 \mathrm{~dB}$ \\
\hline Power Measurement Error & $<1 \%$ & $<1 \%$ \\
\hline Fibre Length & $12 \mathrm{~m}$ & $12 \mathrm{~m}$ \\
\hline Beam Properties & speckle-free, coherence $<0.1$ & speckle-free, contrast $<0.1$ \\
\hline Polarisation & - & $\mathrm{DOP}<1 \%$ \\
\hline System Properties & ISO8 compatible, moveable, compact & ISO8 compatible, moveable, compact \\
\hline Operation & Fully-automated, hands free, remote & Fully-automated, hands free, remote \\
\hline Lifetime & 20 years & 20 years \\
\hline
\end{tabular}


The laser source requirements for the Sentinel-5/UVNS instrument ${ }^{3,4}$ calibration presented significant engineering challenges compared to previous space instrument calibration sources developed by M Squared. To successfully design and build the S5 laser sources involved a large team of 40+ specialists of different disciplines from across the company, as well as consultation with experts at the UK National Physical Laboratory (NPL) and close collaboration with LusoSpace, Airbus DS, TNO and ESA.

\section{SOURCES FOR SPECTRAL CALIBRATION}

The process of identifying an appropriate source for achieving every requirement identified in Table 1, and capable of gapless, narrow linewidth, rapid-tuning, low-noise, ultra-stable and long-lifetimes instantly rules out candidates including dye lasers, optical parametric oscillators, pulsed lasers and diode lasers. ${ }^{5}$ In contrast, M Squared's SolsTiS platform is the world's most functionally advanced, highest specification, fastest tuning and most compact continuous wave (CW) Ti:sapphire laser. SolsTiS is the only fully-automated, hands-free $\mathrm{CW}$ Ti:sapphire laser in the world that can operate in the wavelength range between $670-1100 \mathrm{~nm}$, which can be expanded through M Squared's unique, proprietary, automated frequency extension modules to readily access $260-2700 \mathrm{~nm}$.

In 2019 M Squared officially launched Equinox, our diode-pumped solid state $532 \mathrm{~nm}$ pump laser for the SolsTiS platform. Equinox is one of the narrowest linewidth high-power single-frequency lasers of its class, $<1 \mathrm{MHz}$, making it also inherently stable, a critical property for applications that demand reliable and ultrastable long-term operation.

M Squared's SolsTiS EMM is an external mixing module that offers fully automated tuning in the visible and SWIR wavebands. Access to the visible band is achieved via sum-frequency mixing the SolsTiS output with a dedicated ultra-stable mixing laser (e.g. $1550 \mathrm{~nm}$ or $1950 \mathrm{~nm}$ ), providing up to $150 \mathrm{~nm}$ automated wavelength tuning. The SWIR waveband is accessed via difference frequency mixing the SolsTiS output with a dedicated ultra-stable mixing laser (e.g. $532 \mathrm{~nm}$ or $1550 \mathrm{~nm}$ ), providing up to $1200 \mathrm{~nm}$ automated wavelength tuning.

M Squared's recent innovation, SolsTiS Doublet (previously referred to as ECD-A), is a fully-automated, self-aligning, sealed, high-power SHG cavity capable of rapid and precise, broad wavelength tuning and scanning in the UV and Visible up to $150 \mathrm{~nm}$.

\section{SOURCE DESIGN}

The development program for S5 required a separate assembly, integration and test (AIT) Laser System for to support the calibration of key OGSE hardware. The AIT Laser System was the first of three sources developed over a period of 8 months. The C\&C Lasers are a further two fully enclosed automated widely-tunable, narrowlinewidth, CW laser systems required for spectral and radiometric calibration of the telescope spectrometers. The VN Laser System spans a wavelength range of $300-1100 \mathrm{~nm}$, and the US Laser System spans $260-300 \mathrm{~nm}$ and $685-2700 \mathrm{~nm}$.

\subsection{AIT Laser System}

The AIT Laser System design, shown in Figure 1 and 2, comprises of two Equinox pump lasers (532 nm) (one active, one hot-swap redundant), a custom SolsTiS laser (685-1000 nm), an EMM-1550 (520-600 nm), an ECDQ-A (260-300 nm), an EMM-532-A (300-347 nm) and an ECD-A (347-500 nm). Four specifically-designed beam management modules (BMM) are used to provide necessary power control, power readout, spectral purification and output fibre launch. Four output channels are delivered via four $12 \mathrm{~m}$ length multimode fibres for convenient integration. Furthermore all optical fibres are routed through a custom-built despeckler module to reduce the coherence of light and provide a speckle-free circular output beam profile.

The user can select to utilise either Equinox 1 or Equinox 2 for pumping the SolsTiS laser. The AIT Laser System was designed to incorporate a hot-swappable redundant pump to reduce interruption time in the event of the active Equinox experiencing any issues during test campaigns. A system of waveplates (WP1, WP2, WP3, WP4) and polarising beam splitters provides a variable pump pick-off (VPPO) and set of variable infrared pickoff's (VIRPO1, VIRPO2, VIRPO3) for automated routing of the Equinox and SolsTiS laser light into respective laser modules and beam management modules. 


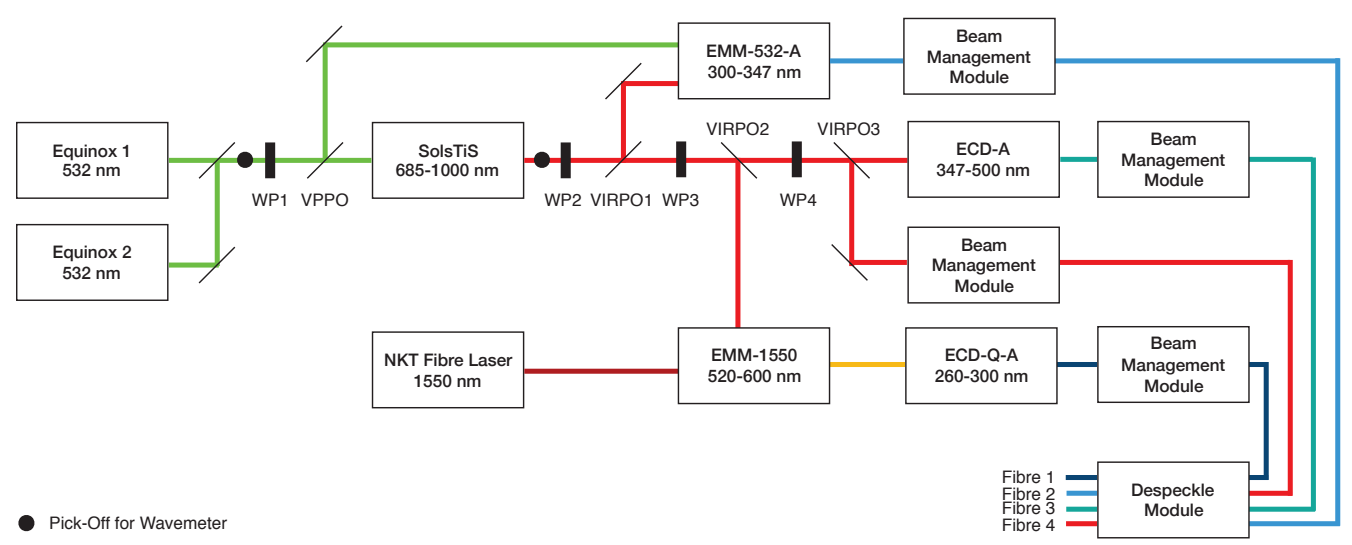

Figure 1. Schematic diagram of the AIT Laser System design. WP: waveplate, VPPO: variable pump pick-off, VIRPO: variable infrared pick-off.
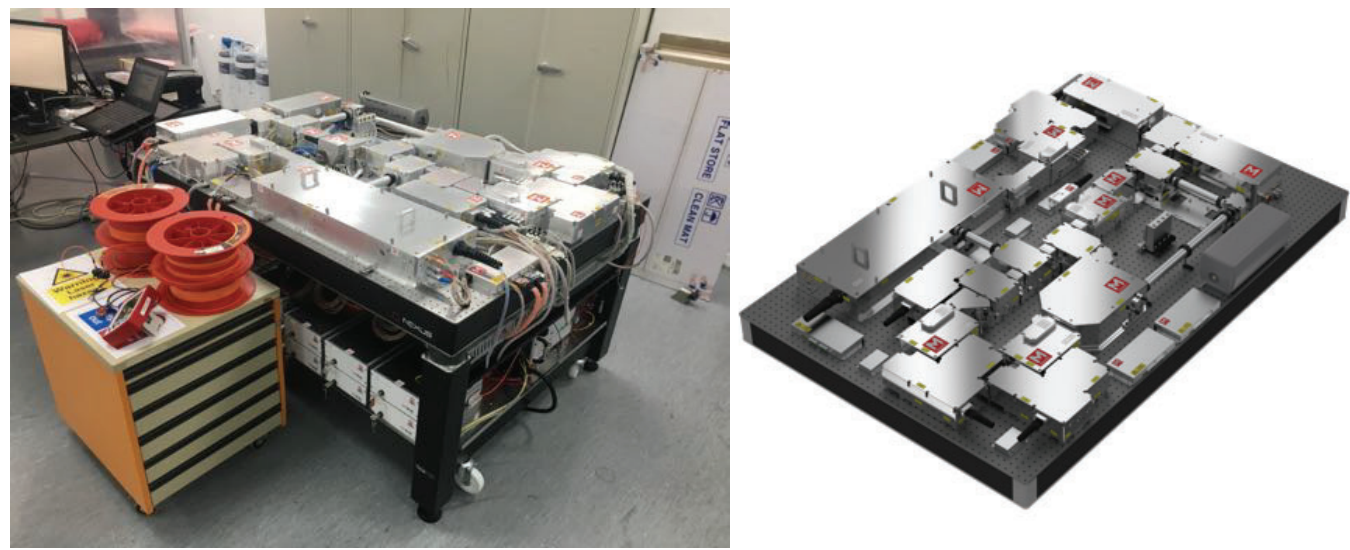

Figure 2. Photograph and rendered drawing of the AIT Laser System.

The EMM-532-A utilises the SolsTiS output and a portion of the Equinox output, achieved by splitting with WP1 and VPPO, to enable sum-frequency mixing in the BBO crystal. The BBO crystal is positioned inside a bow-tie ring cavity which enhances the efficiency of the conversion process. The ICE-Bloc automatically synchronises the orientation of the BBO crystal with the wavelength of the SolsTiS beam so that phase-matching occurs for the selected output wavelength.

The output of the EMM-532-A undergoes subsequent beam pointing correction, spectral purity measures and power measurement and control within the beam management module. The rotation of the BBO crystal causes the beam to be deflected; a Brewster plate, directly following the crystal, automatically counter-rotates relative to the BBO crystal to ensure that the transmitted beam remains un-deflected across the whole wavelength range of the EMM-532-A module.

The ECD-A utilises the SolsTiS output via orientation of WP2, WP3 and WP4 to enable second-harmonic generation in the BBO crystal. Similar to the EMM-532-A, the BBO crystal is positioned inside a bow-tie ring cavity which enhances the efficiency of the conversion process. The ICE-Bloc automatically synchronises the orientation of the BBO crystal with the wavelength of the SolsTiS beam so that phase-matching occurs for the selected output wavelength.

The SolsTiS output is accessed directly via orientation of WP2, WP3, WP4 and VIRPO3 is routed into a beam management module which provides power measurement and control. 
The EMM-1550 utilises a dedicated fibre-coupled 1550nm laser and the SolsTiS output routed via orientation of WP2, WP3 and VIRPO2. Beams from the fibre laser and SolsTiS are combined on a dichroic mirror and then undergo a single pass through a PPLN fan grating. The fan grating has a gradient in its poling period across the width of the crystal; phase-matching is achieved by simply translating the PPLN fan transverse to the optical beam to select the period which phase-matches the desired wavelength. The advantage of tuning the phase-matching in this way is that wavelength can be adjusted without any change in the beam alignment. The fibre laser used is a high-power narrow linewidth, ultra-low noise $1550 \mathrm{~nm}$ system available from NKT Photonics (AdjustiK seed and BoostiK amplifier).

The visible light output of the EMM-1550 is mode-matched into the ECD-Q-A, which enables secondharmonic generation in the BBO crystal. Again, the BBO crystal is positioned inside a bow-tie ring cavity which enhances the efficiency of the conversion process. The ICE-Bloc automatically synchronises the orientation of the BBO crystal with the wavelength of the SolsTiS beam so that phase-matching occurs for the selected output wavelength.

The output of the ECD-Q-A undergoes subsequent beam pointing correction, spectral purity measures and power measurement and control within the beam management module. Rotation of the BBO crystal causes the beam to be deflected, hence a Brewster plate is automatically orientated to ensure that the transmitted beam remains un-deflected across the whole wavelength range of the ECD-Q-A module.

The output from each of the beam management modules is fibre coupled via the despeckle module which actively vibrates the optical fibre to reduce the coherence and improve the uniformity of the output beam profile.

\subsection{VN Laser System}

The VN Laser System, shown in Figure 3 and 4, comprises of three Equinox (532 nm), two used as pump lasers (one active $18 \mathrm{~W}$, one hot-swap redundant $18 \mathrm{~W}$ ) and a separate dedicated mixing source (6W). The Equinox light is used to pump one of two SolsTiS lasers $(685-950 \mathrm{~nm}$ and $950-1100 \mathrm{~nm})$. Only one SolsTiS is active at any time depending on the desired output wavelength, and the master control system automates this by adjustment of the motorised waveplate (WP1).

The output of each SolsTiS is combined into a common path within the SolsTiS combiner module using a system of motorised waveplates (WP2, WP3, WP4) and polarising beam splitters. Depending on the output wavelength required, the master control software automatically routes the SolsTiS laser light to four possible output paths by adjusting one or a combination of waveplate angles.

The full SolsTiS tuning range $(685-1100 \mathrm{~nm})$ can thus be accessed directly via fibre 4, or to access the UV or Visible spectral range the SolsTiS light is steered into the appropriate laser module and hence frequency conversion mode to achieve the target wavelength.

The EMM-532-A utilises the SolsTiS output and a dedicated mixing Equinox output to enable sum-frequency mixing in a non-linear crystal as previously described. The output light $(300-347 \mathrm{~nm})$ is coupled into a beam management module for power measurement, control and launching into fibre.

Similarly, the ECD-A utilises the SolsTiS output via orientation of waveplates to enable second-harmonic generation in the non-linear crystal as previously described, providing $>200 \mathrm{~nm}$ across the Visible and UV spectrum. Similarly, the ECD-A output $(347-550 \mathrm{~nm})$ is coupled into a beam management module for power measurement, control and launching into fibre.

The EMM-1950 utilises a dedicated fibre-coupled 1950nm fibre laser and the SolsTiS output undergoing a single pass through a PPLN fan grating to generate visible light $(550-685 \mathrm{~nm})$ via sum frequency mixing. The fibre laser used is a high-power narrow linewidth, low-noise $1950 \mathrm{~nm}$ system available from AdValue. The visible output is steered into a beam management module for power measurement and control.

A small fraction of the visible light output of the EMM-1950 picked-off to an optical fibre to allow direct access by a wavemeter integrated within the system. As well as directly monitoring the output of the EMM-1950, the wavemeter has a four-channel switch to allow for monitoring the two SolsTIS outputs and the dedicated mixing Equinox output also, thereby providing a means for direct or inferred wavelength measurement across the full spectral range of the system. 
The output light from each BMM is routed to a despeckler module to actively oscillate a number of multimode fibre coils in order to sufficiently mix the mode transmission of the propagating light within a specific duration. The resulting output is a uniform Gaussian beam profile.

Affixed to the inside of the support frame is located a four-channel fibre connector bracket and a 2 channel fibre splitter to allow up to four separate $12 \mathrm{~m}$ length and an $8 \mathrm{~m}$ length optical fibre to be connected for convenient delivery to the customer application.

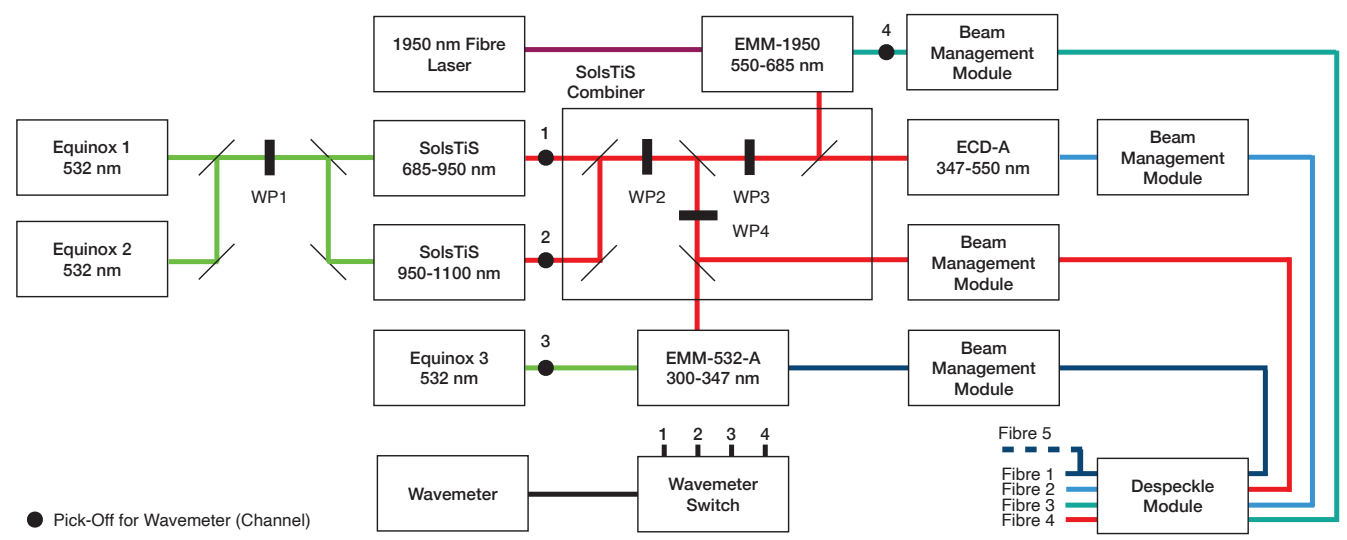

Figure 3. Schematic diagram of the VN Laser System design. WP: waveplate, VPPO: variable pump pick-off, VIRPO: variable infrared pick-off.
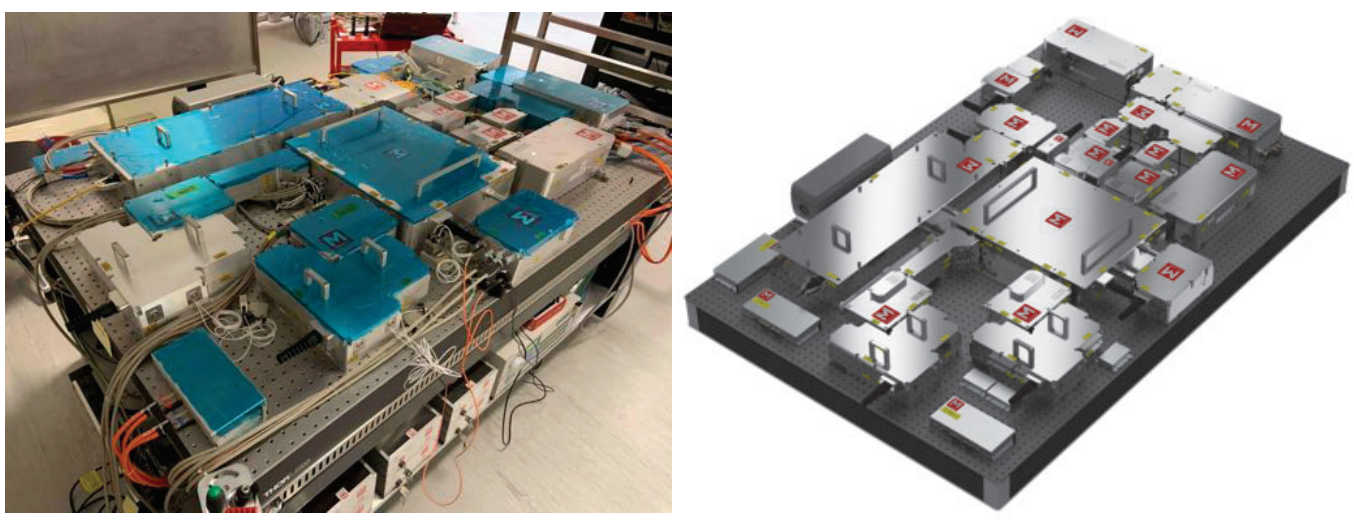

Figure 4. Photograph and rendered drawing of the VN Laser System.

\subsection{US Laser System}

The US Laser System, shown in Figure 5 and 6, comprises of two Equinox (532nm) pump lasers (one active $18 \mathrm{~W}$, one hot-swap redundant 18W). Similar to the design of the VN Laser System, the Equinox light is used to pump one of two SolsTiS lasers $(685-950 \mathrm{~nm}$ and $950-1100 \mathrm{~nm})$. A portion of the Equinox light can also be steered to a separate optical path for frequency mixing.

The output of each SolsTiS is combined into a common path within the SolsTiS combiner module using a system of motorised waveplates (WP3, WP4, WP5) and polarising beam splitters. Depending on the output wavelength required, the master control software automatically routes the SolsTiS laser light to four possible output paths, by adjusting one or a combination of waveplate angles. The full SolsTiS tuning range $(685-$ $1100 \mathrm{~nm}$ ) can be accessed directly or steered into appropriate laser module for frequency conversion to reach UV or SWIR wavebands. 
As described previously, the EMM-1550 utilises a dedicated fibre-coupled 1550nm fibre laser and the SolsTiS output for the generation of $>1 \mathrm{~W}$ of visible light $(520-600 \mathrm{~nm})$. Again, the visible output from the EMM-1550 is frequency doubled in a non-linear crystal to access the UV band $(260-300 \mathrm{~nm})$ and the output fibre coupled via the beam management module.

The EMM-532-DFG provides short-wave infrared (SWIR) light $(1100-2000 \mathrm{~nm})$ by difference frequency mixing a portion of the Equinox light and the SolsTiS light. The SWIR output is steered into an output telescope which also provides direct access to wavemeter pick-off using a small fraction of the light. The SWIR output from the telescope is steered into a beam management module.

Similarly, the EMM-1550-DFG outputs SWIR light $(2000-2700 \mathrm{~nm})$ by difference frequency mixing a dedicated $1550 \mathrm{~nm}$ fibre laser and the SolsTiS light.

A high-accuracy wavemeter (sensitive to IR wavelengths) and eight-channel switch is integrated within the system design to allow monitoring of both SolsTiS outputs, the EMM-532-DFG output, the 1550nm fibre laser and a stabilised reference laser $(1532 \mathrm{~nm})$, thereby providing a means for direct or inferred wavelength measurement across the full spectral range of the system.

The four outputs from each of the beam management modules is fibre coupled via the despeckle module which actively vibrates the optical fibre to reduce the coherence and improve the uniformity of the output beam profile.

A four-channel fibre connector bracket is mounted to the support frame to provide a convenient means for attaching up to four optical fibres of length $8 \mathrm{~m}$.

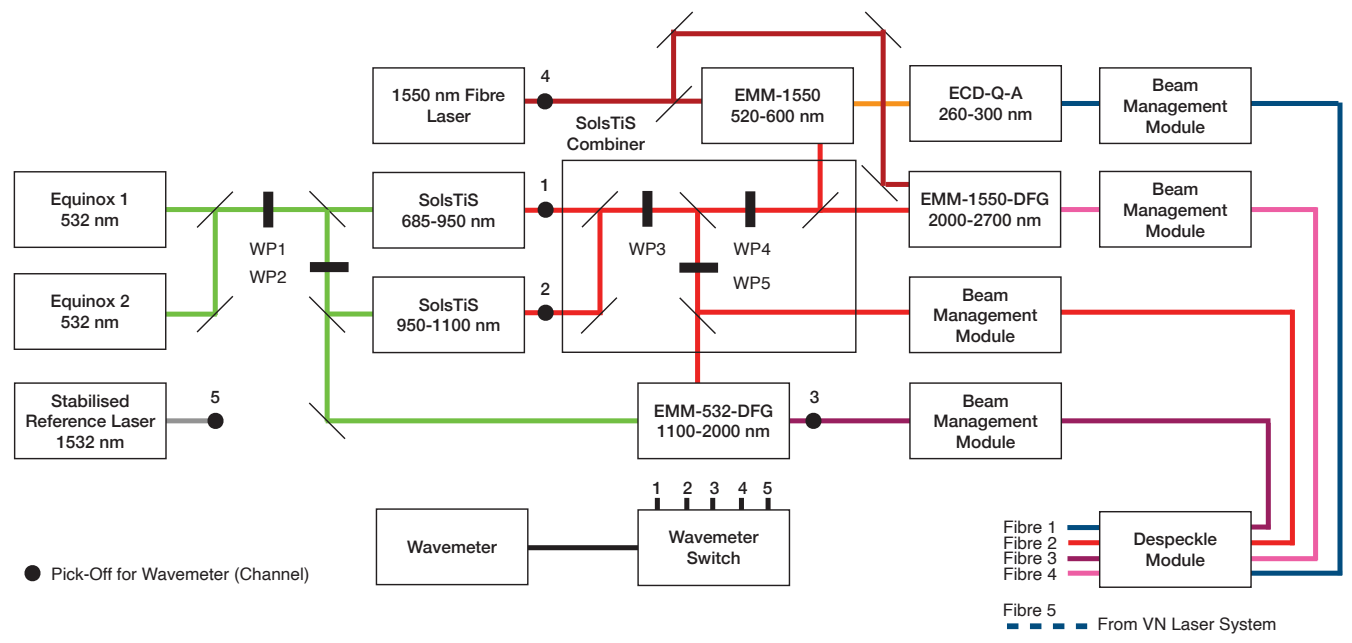

Figure 5. Schematic diagram of the US Laser System design. WP: waveplate, VPPO: variable pump pick-off, VIRPO: variable infrared pick-off.

\section{OUTPUT BEAM MANAGEMENT}

\subsection{Power Control, Power Stabilisation and Power Readout}

The SolsTiS laser platform offered many of the important features required such as ultra-stable, narrow-linewidth, high-accuracy, rapid and gapless tuning from UV to SWIR. However there were many more laser requirements that required significant engineering efforts such as high-dynamic range automated power control, high-accuracy power readout (relative to the fibre output), enhanced spectral purification, self-optimised fibre coupling, longterm active power stabilisation, de-speckling provision.

To achieve these performance criteria, M Squared Lasers have developed an external module to be used at the output of all laser modules for automated hands-free control of the output power, real-time power measurement, 

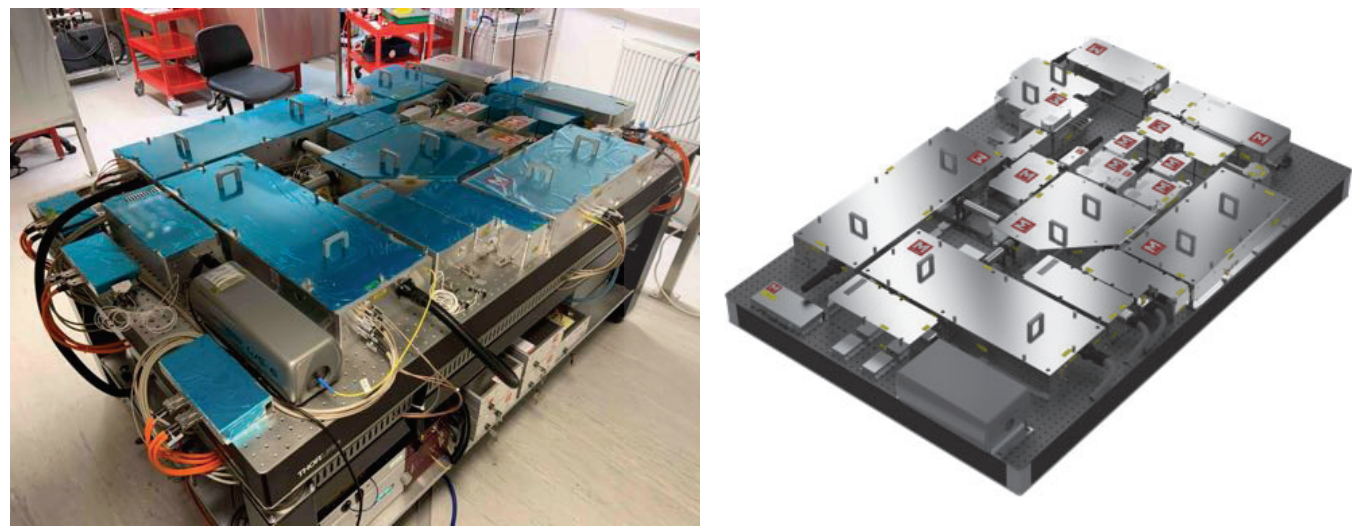

Figure 6. Photograph and rendered drawing of the US Laser System.

active power stabilisation and efficient optical fibre coupling. This technology resulted from a collaboration with NPL and extensive design, development, prototyping, characterisation and testing. The final design provides a flexible solution suitable for customising with specific optic sets appropriate for all spectral bands.

The Beam Management Module (BMM), shown in Figure 7, uses a system of motorised variable and stepped attenuation optics for providing high dynamic range continuous power control from $100 \%$ to less than $0.0001 \%$ (up to $60 \mathrm{~dB}$ ). A small portion of the beam is picked off at two locations prior to fibre coupling to provide realtime power measurement (at 1 second intervals) that can be calibrated using a commercially available calibrated power meter to provide accurate absolute power diagnostics relative to the fibre output. The optical design of the BMM further provides option for active power stabilisation to compensate for short-term fluctuations and long-term power drifts.

Evaluating the total transmission properties of the SolsTiS BMM design shows $52-60 \%$ across $685-1100 \mathrm{~nm}$ tuning range, which includes the fibre coupling efficiency. The same analysis of the automated ECD BMM design, which incorporates more optical elements, shows overall transmission of $26-33 \%$ across $260-300 \mathrm{~nm}$, including the fibre coupling losses.
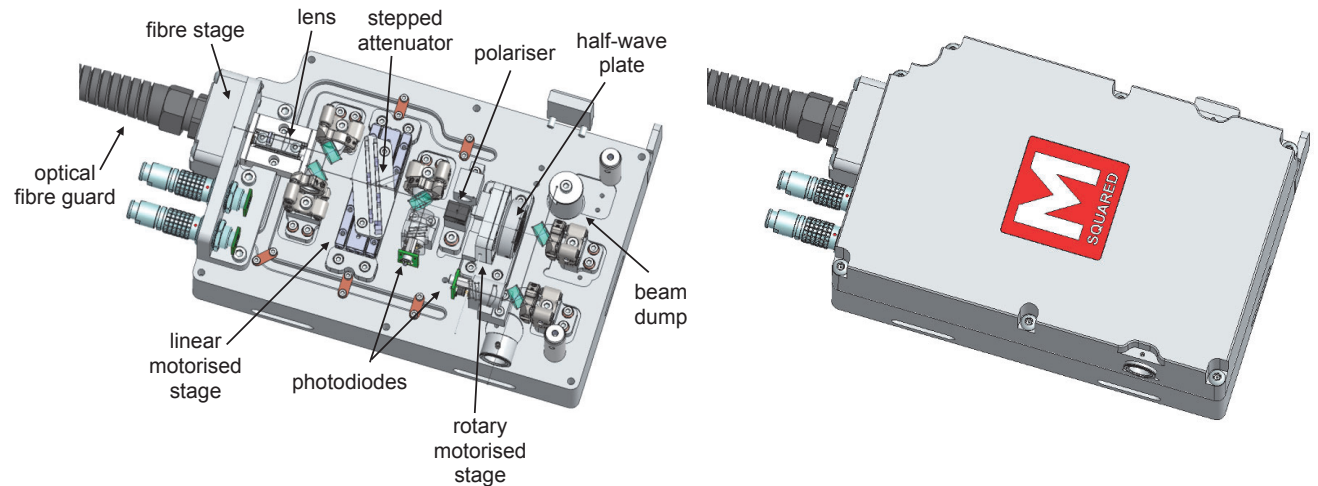

Figure 7. Mechanical drawings of the SolsTiS Beam Management Module.

Another design of the BMM for the automated frequency doubling and sum frequency mixing modules, shown in Figure 8, incorporates a pair of optical prisms to isolate trace fundamental and mixing inputs from the output beam, thus ensuring spectral purity requirements are met across all wavebands. 

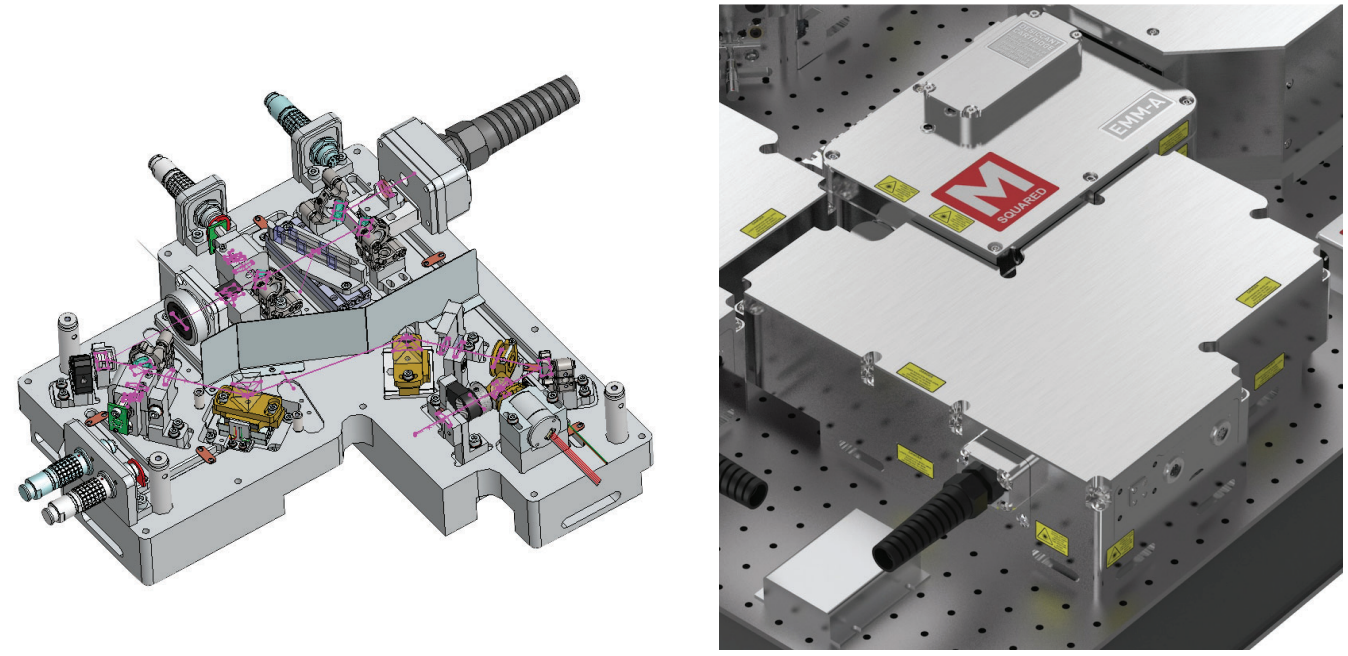

Figure 8. Mechanical drawing (left) and render (right) of the beam management module for automated ECD and EMM laser modules.

\subsection{Despeckler Module}

The choice for optical fibre delivery from each laser module to the OGSE provides convenient interfacing and safety however also introduces another challenge of reducing the high-contrast speckle pattern in the output beam. The transmission of single-wavelength, highly-coherent, light through fibre inherently leads to speckle due to interference of a large number of highly-coherent optical wavefronts. One strategy to reduce the speckle relies on illuminating a rotating diffuser at a significantly faster rate than the integration time of the measurement, however this device must be incorporated at the fibre output, typically inside the design of an integrating sphere.

M Squared Lasers worked in collaboration with NPL, to design, build and test a fibre-based despeckler module, which vibrates of the fibre on short timescales to mix the spatial modes, thus reducing the coherence and inhomogeneity of the observed speckle at a longer timescale. ${ }^{6}$

The design of the Despeckler Module, shown in Figure 9, utilises an electroacoustic transducer and a rigid mechanical support frame to actively oscillate a number of multimode fibre coils in order to sufficiently mix the mode transmission of the propagating light, when evaluated over a specific duration, typically 1 second. The Despeckler module can be remotely controlled and activated automatically by the master control software for the laser system.
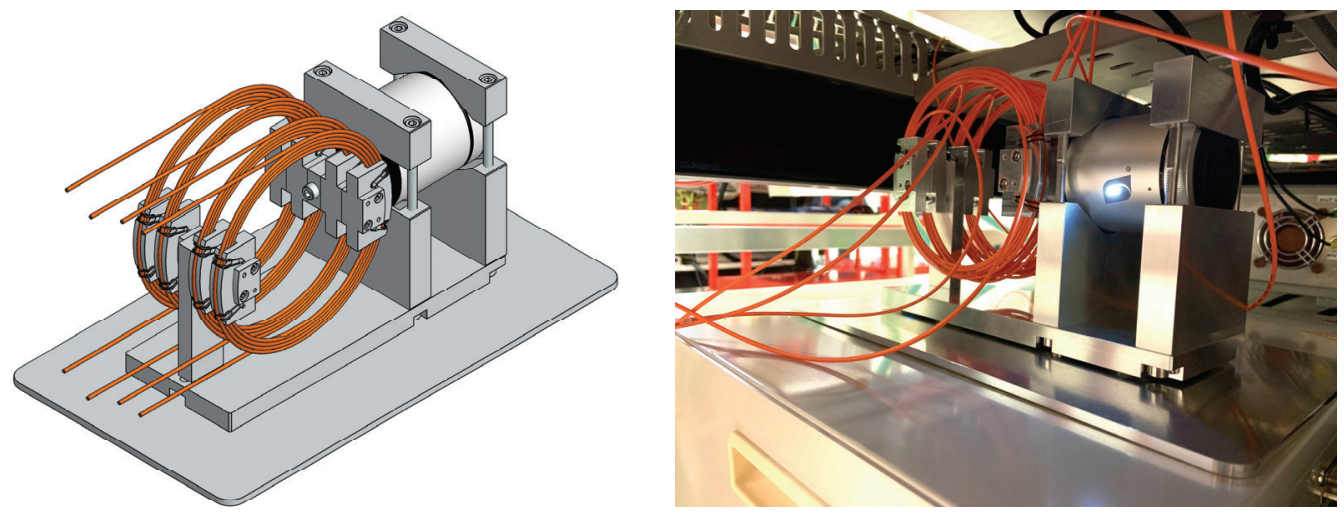

Figure 9. Mechanical drawing (left) and photo (right) of the despeckler module integrated to the design of AIT, VN and US laser systems, optimised to reduce the speckle from four optical fibres. 


\section{LASER PERFORMANCE}

The AIT Laser System was installed at the Airbus facility in Germany mid 2019, with the VN and US Laser Systems awaiting installation at Rutherford Appleton Laboratory (RAL) in the UK, expected to be late 2021. Here we present a sample of the key performance data from each of the laser systems.

\subsection{Broad Wavelength Tuning}

In one test, the VN and US Laser Systems were continuously wavelength stepped, with a step size of $1 \mathrm{~nm}$ in band and $5 \mathrm{~nm}$ out of band across the entire tuning range. Both laser systems were remotely controlled and operated in parallel, precisely how the lasers will be used during extensive spectral and radiometric calibration test campaigns. A summary of the results is provided in Table 2 and Figure 10 and Figure 11.

Table 2. Results of Stray Irradiance laser test performed on US and VN Laser Systems.

\begin{tabular}{|l|l|l|}
\hline Parameter & VN Laser System & US Laser System \\
\hline Wavelength Steps & 391 & 569 \\
\hline Test Duration & $157 \mathrm{~min}$ & $235 \mathrm{~min}$ \\
\hline Average Tune Time & $24 \mathrm{~s}$ & $25 \mathrm{~s}$ \\
\hline
\end{tabular}

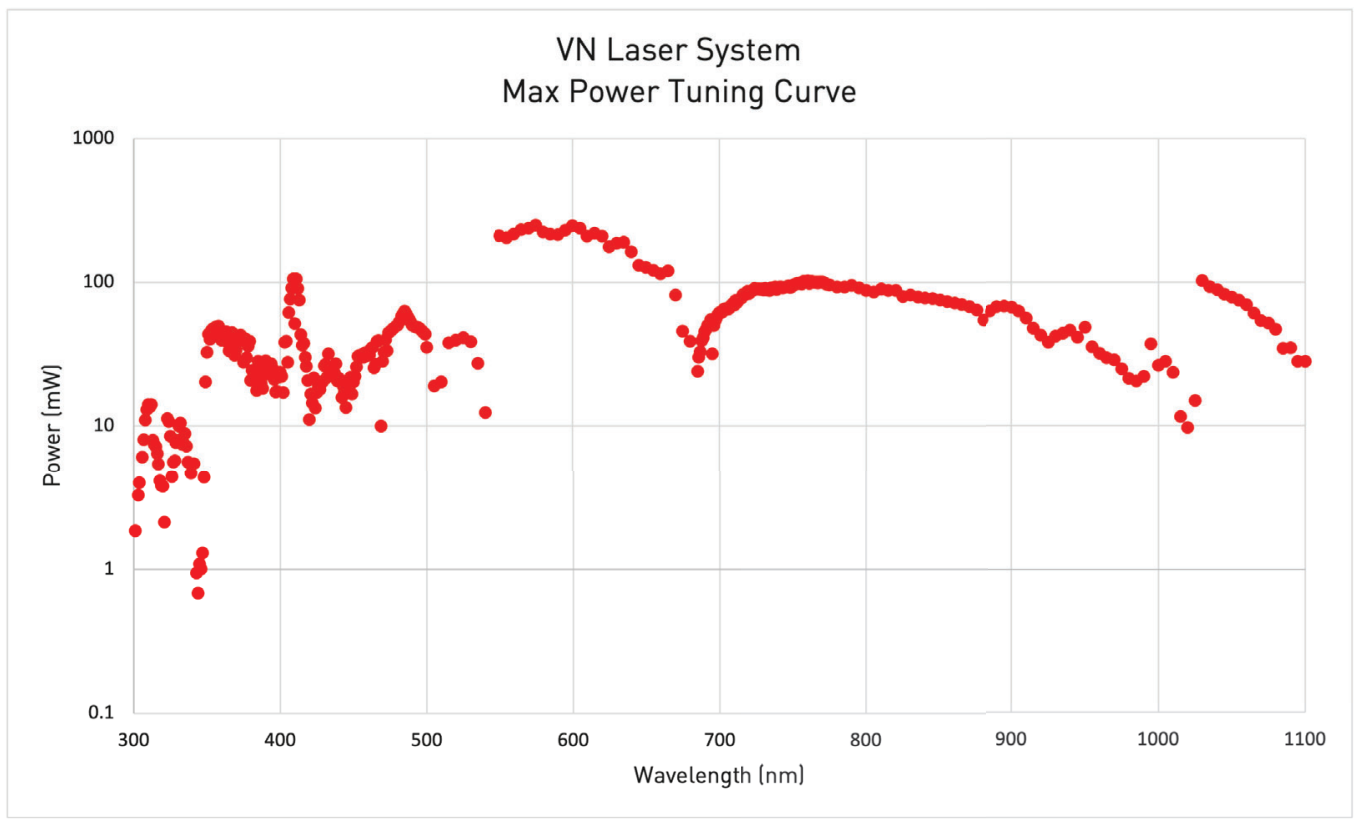

Figure 10. Maximum output power measured across the full tuning range on the VN Laser System calibrated to the end of $12 \mathrm{~m}$ optical fibres, as measured during extensive factory acceptance testing.

\subsection{High Dynamic Range Power Control}

We can evaluate the dynamic range of the power control elements incorporated within the beam management module by scanning the half-wave plate from maximum to minimum position while adjusting the stepped attenuator across the beam. For a specified wavelength the associated optical fibre was coupled to the sensor head such that the beam underfills the active area of the sensor. Measurements from the power meter were recorded by computer while the waveplate was rotated. The measurement was repeated for each position of the ND filter used to achieve the appropriate attenuation factor. The result of this test at $450 \mathrm{~nm}$ is shown in Figure 12. Here we demonstrate up to $60 \mathrm{~dB}$ attenuation is available. 


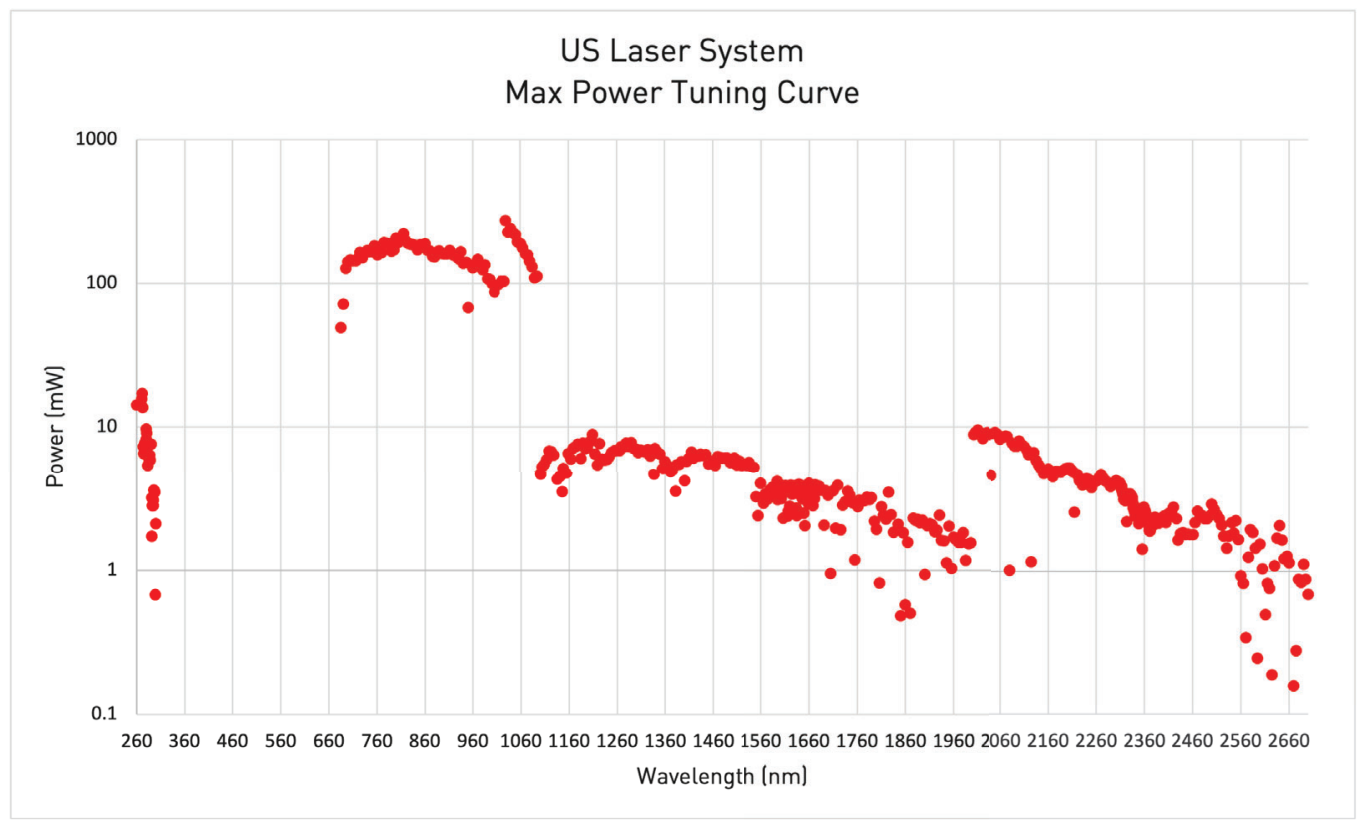

Figure 11. Maximum output power measured across the full tuning range on the US Laser System calibrated to the end of $8 \mathrm{~m}$ optical fibres, as measured during extensive factory acceptance testing.

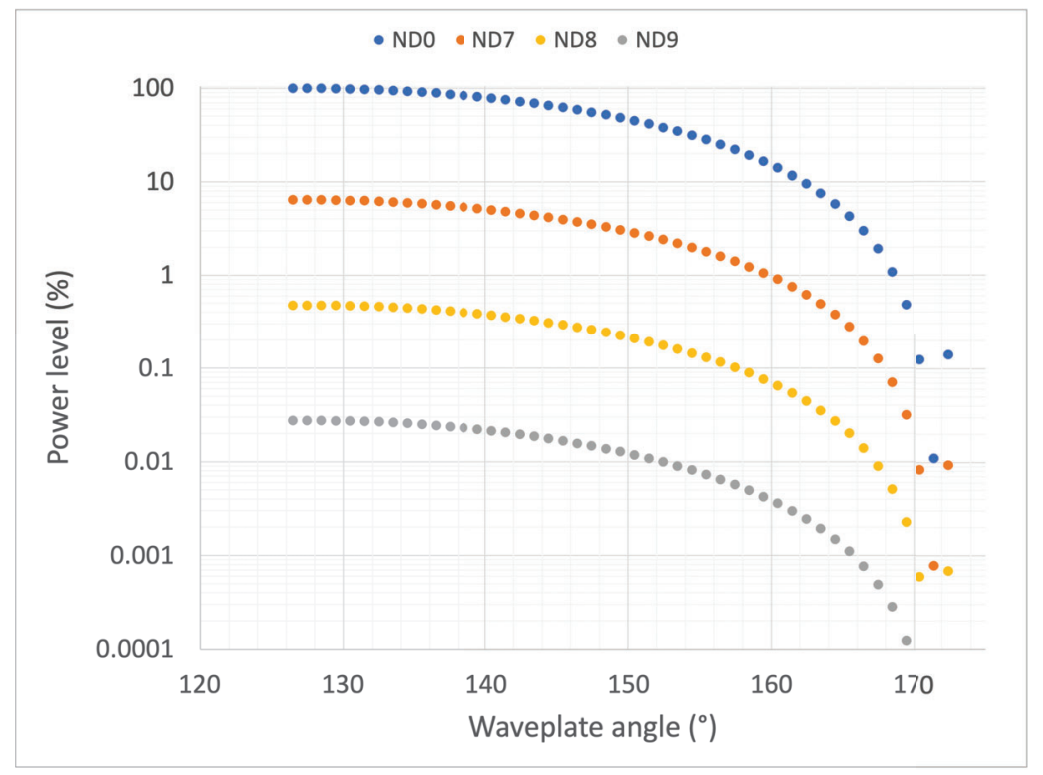

Figure 12. Measured relative output power at $450 \mathrm{~nm}$ as a function of waveplate angle and stepped attenuation, exhibiting up to $60 \mathrm{~dB}$ power control.

\subsection{Despeckler}

To evaluate the efficiency of the Despeckle Module a test can be performed to assess the output beam profile. This test involves positioning the output beam at a distance that expands the profile until it under-fills a focal plane array sensor with appropriate resolution to resolve the speckle features, and the data subsequently analysed using image processing software. The broad spectral coverage of the laser sources $260-2700 \mathrm{~nm}$ requires the use of a pyroelectric imager or CMOS camera.

In order to fully resolve the speckle from the fibre, the imaging system needs to resolve details of the smallest 
speckle. The size of the speckle grains is inversely proportional to the number of transverse modes supported by the fibre, which in turn increases with fibre mode size and NA. The smallest speckle grain will be the size of the smallest spot achievable with the NA available, and is given by the Abbe limited spot size. This spot size will be of order a few wavelengths, facilitating the need to magnify the beam between the fibre and the camera. The speckle size will increase upon propagation directly proportional to the beam size, as the number of speckle grains remains constant. The required beam magnification is that which magnifies the smallest speckle grain such that it is larger than a single pixel of the camera, which was calculated. If this magnification level also fits the entire beam onto the camera area then the beam and its speckle are fully resolved, which was confirmed for all fibre outputs.

The test method is therefore to position the camera at a distance from the fibre output such that the beam slightly under-fills the camera, to ensure that the speckle will be suitably resolved while imaging the entire beam. The laser output power and camera exposure time are set so as to prevent saturation of the sensor. A time averaged image over a duration of 1 second is recorded on computer using the camera control software. An 'off-image' and 'on-image' are obtained when the Despeckler Module is turned off and on respectively. The mean speckle size shall is evaluated according to the normalized autocorrelation function of the off-image. A cross section along one axis through the centre of the beam from both images is obtained and a region of interest with a size 10x the mean speckle size is be defined in both images for evaluating the speckle contrast ratio. We define the contrast ratio $\mathrm{C}$ according to:

$$
C=\frac{I_{\max }-I_{\min }}{I_{\max }+I_{\min }},
$$

where $I_{\max }$ and $I_{\min }$ are the maximum and minimum intensity respectively. The contrast ratio is evaluated along both the $\mathrm{x}$ and $\mathrm{y}$ axes cross-sections and the average contrast is determined. Example results of this test performed at 301nm is provided in Figure 13. The left image is obtained with the Despeckler off and the right image is obtained with the Despeckler on with a vibration frequency of $\sim 60 \mathrm{~Hz}$, indicating a contrast ratio of $C=0.066$ with the Despeckler module active.
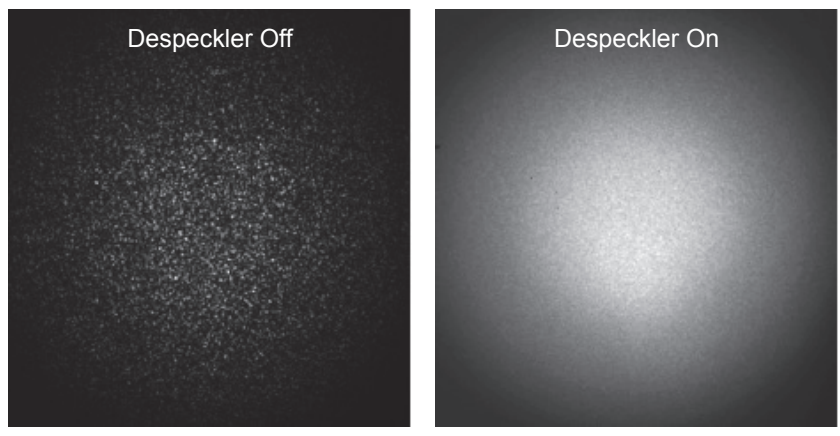

Figure 13. Images of the fibre output acquired when the Despeckler Module is off (left) and on (right).

\subsection{Wavelength Accuracy}

The absolute wavelength accuracy of the laser modules is evaluated using dedicated wavelength meters integrated into the system design, measuring at specific pick-off points on each table. These pick-off points provide direct measurement of laser light, however in cases where direct measurement is not possible due to limited wavemeter spectral responsivity, it is still possible to attain indirect measurements of wavelength.

The frequency conversion process of non-linear media, such as beta barium borate (BBO) used in ECD and EMM modules, is well understood. ${ }^{7}$ At any instant, the generated frequency $\nu_{\text {out }}$ is twice the incident frequency $\nu_{i n}$ :

$$
\nu_{\text {out }}=2 \nu_{\text {in }},
$$


and similarly, the linewidth of the output light can be calculated according to:

$$
\Delta \nu_{\text {out }}=2 \Delta \nu_{\text {in }}
$$

Similarly well understood processes are used for both sum and difference frequency mixing. These are:

$$
\begin{aligned}
& \nu_{\text {out }+}=\nu_{1}+\nu_{2}, \\
& \nu_{\text {out }-}=\nu_{1}-\nu_{2},
\end{aligned}
$$

where $\nu_{\text {out }}$ and $\nu_{\text {out }}$ are the result of sum and difference frequency mixing respectively, and $\nu_{1}$ and $\nu_{2}$ are the frequencies of the two lasers which are mixed. It is also possible to cascade the frequency doubling process, in which case

$$
\nu_{\text {out }}=4 \nu_{\text {in }}
$$

In summary the laser systems are designed to utilise a mix of direct and indirect measurements of wavelength.

The target wavelength $\left(\lambda_{\text {tar }}\right)$ will be compared to the measured wavelength $\left(\lambda_{\text {meas }}\right)$ at samples across all wavebands and the wavelength accuracy calculated, defined as:

$$
\lambda_{a c c}=\sqrt{\left(\lambda_{\text {tar }}-\lambda_{\text {meas }}\right)^{2}} .
$$

The wavelength accuracy results for all wavebands, obtained from the VN and US Laser Systems, are presented in Figure 14. In one instance during this test the wavelength at $340 \mathrm{~nm}$ failed to tune due to a water absorption feature, however all other wavelengths requested exhibited an accuracy that considerably exceeded the specification, determined by the accuracy of the associated wavemeter.

Wavelength Accuracy

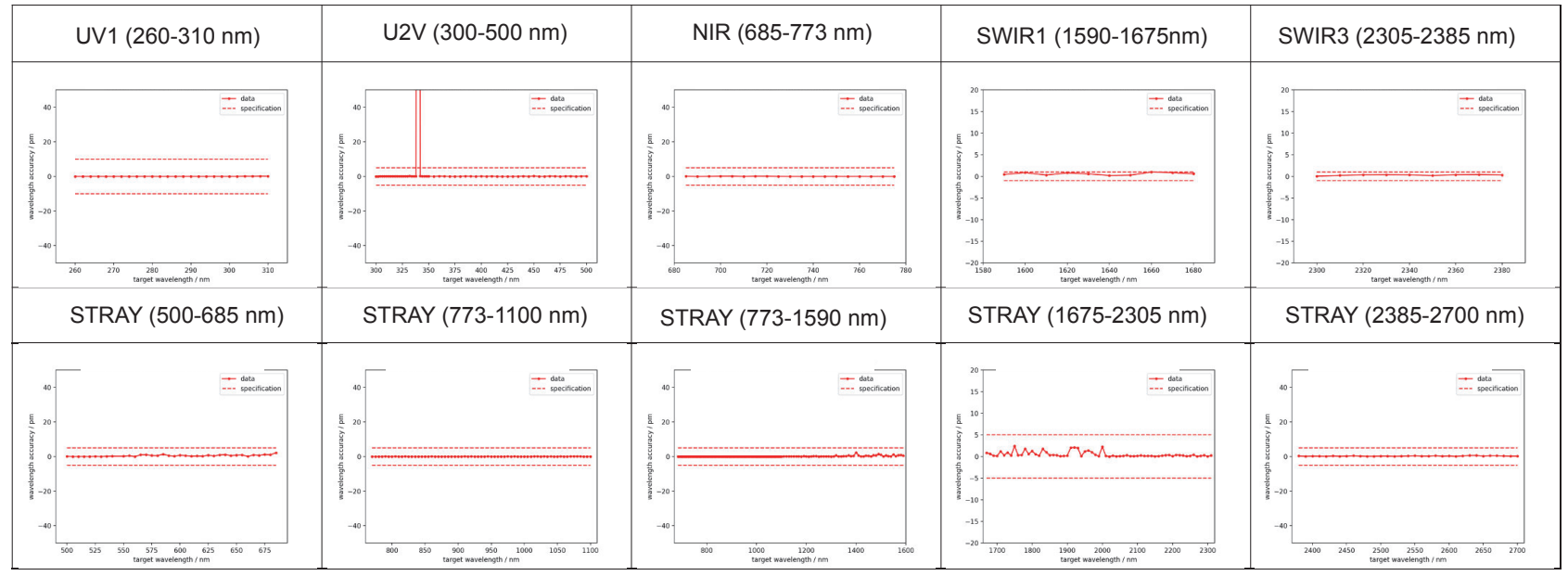

Figure 14. Wavelength accuracy measurements obtained from the VN and US Laser Systems. 


\subsection{Power Stabilisation}

The continuous power control elements within each BMM allow for the implementation of active feedback on all output channels to maintain a target power, minimise short-term fluctuations as well as long term drifts. To assess the long-term power stability the output of the fibre is coupled to an external power meter sensor positioned at a distance such that the expanding beam underfills the active area of the sensor head. For each wavelength tested, the power was monitored at intervals of 1s with 1s integration time for a duration of 24 hours. From the acquired data the percentage RMS error of the intensity is calculated according to the standard deviation of the signals divided by the mean intensity. Figure 15 shows the measured power stability over a 24 hour period at an output wavelength of $1550 \mathrm{~nm}$ from the EMM-532-DFG laser module.

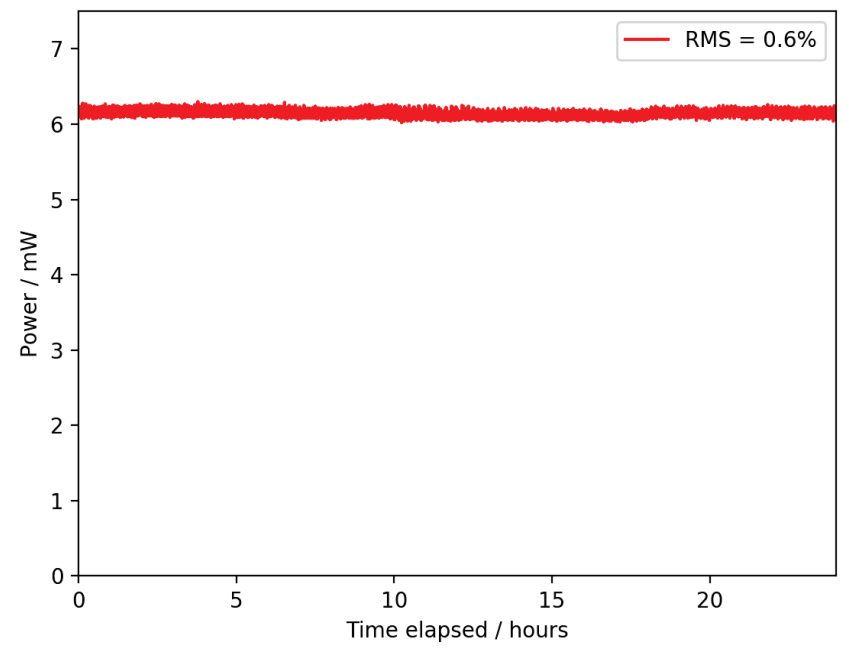

Figure 15. Measured power stability over 24hrs at an output wavelength of 1550nm from the EMM-532-DFG laser module.

\section{SOFTWARE CONTROL}

Remote control of the AIT, US and VN Laser System is provided by a list of TCP/IP commands over Ethernet to a local dedicated laptop which is connected to all control equipment. A custom software application was developed, an example is shown in Figure 16, which provides convenient, simple and user-friendly, master control of the entire laser system.

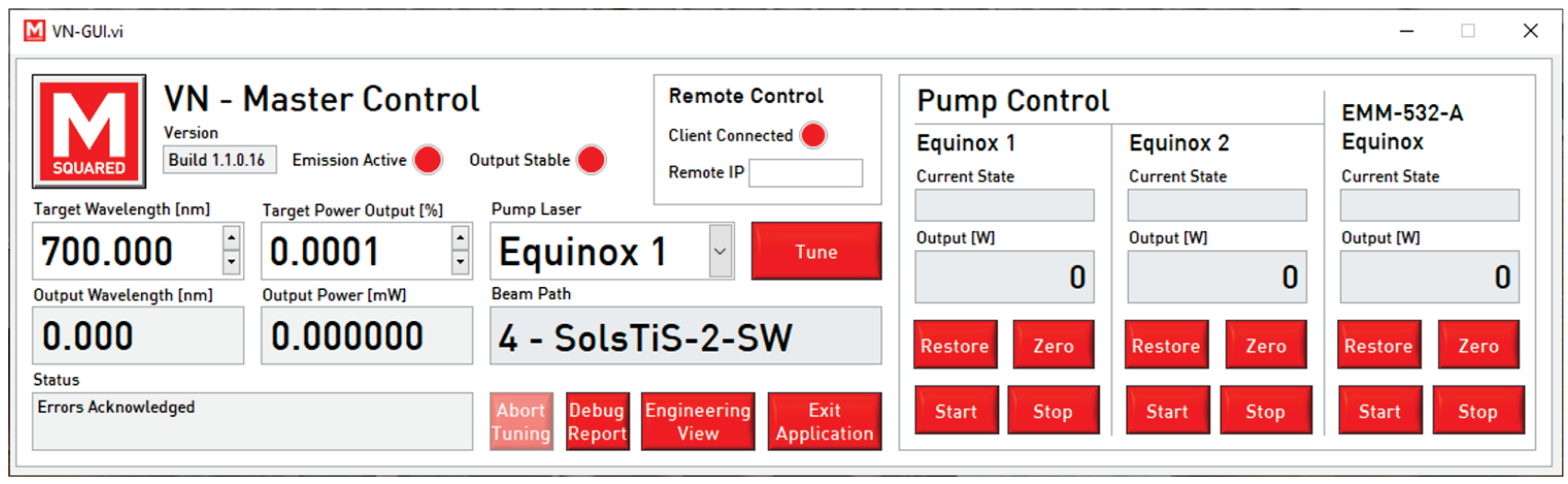

Figure 16. Example of the master control interface provided for each laser system, giving full access to wavelength, relative power, absolute power, power readout among many more features. 


\section{CONCLUSIONS AND NEXT STEPS}

M Squared Lasers have successfully designed, developed and tested three uniquely advanced, fully-automated, remotely-operated, narrow-linewidth laser systems, providing rapid and gapless wavelength tuning from 260 $2700 \mathrm{~nm}$. Coupled with state-of-the-art output beam management and diagnostics, these laser systems represent a technological breakthrough for enabling important spectral and radiometric calibration of the imaging spectrometers that will soon be launched on-board the Sentinel-5 satellite. The technological advances made on these systems will further benefit future calibration sources for space and scientific instrumentation.

\section{ACKNOWLEDGMENTS}

M.E. and J.N. gratefully acknowledge the valuable advice and support provided by Nigel Fox and Paul Green at NPL towards the design principles or the power control and depeckler module. The authors gratefully acknowledge the valuable input from the entire engineering team at M Squared towards the development and testing of the AIT and C\&C laser systems. M.E., J.N., G.M. and G.M. further acknowledge the collaborative approach between M2, LS, ADS, TNO and ESA throughout the laser development.

\section{REFERENCES}

[1] Bauwens, M., Compernolle, S., Stavrakou, T., Müller, J.-F., Van Gent, J., Eskes, H., Levelt, P. F., van der A, R., Veefkind, J., Vlietinck, J., et al., "Impact of coronavirus outbreak on no2 pollution assessed using tropomi and omi observations," Geophysical Research Letters 47(11), e2020GL087978 (2020).

[2] Aschbacher, J., "Esa's earth observation strategy and copernicus," in [Satellite earth observations and their impact on society and policy], 81-86, Springer, Singapore (2017).

[3] Albiñana, A. P., Erdmann, M., Wright, N., Martin, D., Melf, M., Bartsch, P., and Seefelder, W., "Sentinel-5: the new generation european operational atmospheric chemistry mission in polar orbit," in [Infrared Remote Sensing and Instrumentation XXV], 10403, 104030P, International Society for Optics and Photonics (2017).

[4] Irizar, J., Melf, M., Bartsch, P., Koehler, J., Weiss, S., Greinacher, R., Erdmann, M., Kirschner, V., Albinana, A. P., and Martin, D., "Sentinel-5/uvns," in [International Conference on Space Optics-ICSO 2018], 11180, 1118004, International Society for Optics and Photonics (2019).

[5] Hecht, E., [Optics], Pearson Education (2017).

[6] Zajiczek, L., Winkler, R., Hobson, T., Green, P., and Fox, N., "Prototype of cryogenic solar absolute radiometer and transfer radiometer for on-board calibration of spectral earth imager," Journal of Physics: Conference Series 972, 012005 (feb 2018).

[7] Boyd, R. W., [Nonlinear optics], Academic press (2020). 